\title{
Estratégia no monitoramento de pa- cientes suspeitos de Covid-19 em um município do sul do Brasil
}

\author{
Strategy for monitoring suspected Covid-19 in a \\ municipality of the south of Brazil
}

Paula Souza de Freitas ${ }^{1}$, Carlos Eduardo Coradassi ${ }^{2}$

1. ORCID: http://orcid.org/0000-0002-4416-5291. Mestrado. Prefeitura Municipal de Ponta Grossa, Ponta Grossa, Paraná, Brasil. E-mail: paulasouzadefreitas@yahoo.com.br.

2. ORCID: http://orcid.org/0000-0002-9812-0152. Doutorado. Prefeitura Municipal de Ponta Grossa, Ponta Grossa, Paraná, Brasil. E-mail: coradassi@gmail.com.

CONTATO: Nome do autor correspondente: Paula Souza de Freitas | Endereço: Rua Alberto Divardim, 121, Giana | Telefone: 42-999299353 | E-mail: paulasouzadefreitas@yahoo.com.br

RESUMO A pandemia da Covid-19 tem proporcionado um grande desafio aos gestores municipais no combate ao Coronavírus, onde a Atenção Primária tem papel relevante e estratégico no monitoramento de sintomáticos respiratórios. Trata-se de uma pesquisa com o objetivo de mostrar o processo e o resultado do trabalho desenvolvido pela Atenção Primária no monitoramento de pacientes suspeitos em Ponta Grossa, Paraná de março a maio de 2020. A busca ativa e o monitoramento de sintomáticos respiratórios na prevenção e esclarecimento a respeito da Covid-19 foram essenciais na estratégia, demonstrando para a população o papel da Atenção Primária.

DESCRITORES: Covid-19. Política Pública. Atenção Primária à Saúde. Pandemia. 


\begin{abstract}
The Covid-19 pandemic has provided a great challenge to municipal managers in the fight against Coronavirus, where Primary Care has a relevant and strategic role in monitoring respiratory symptoms. This is a survey with the objective of showing the process and the result of the work developed by Primary Care in monitoring suspected patients in Ponta Grossa, Paraná from March to May 2020. The active search and monitoring of respiratory symptoms in the prevention and clarification regarding Covid-19 were essential in the strategy, demonstrating to the population the role of Primary Care.
\end{abstract}

DESCRIPTORS: Covid-19. Public Policy. Primary Health Care. Pandemics.

\title{
INTRODUÇÃO
}

$\mathbf{U}$

m número crescente de casos de pneumonia causados por uma nova cepa coronavírus da chamada nova Covid-19 foi identificado no início de dezembro de 2019 na cidade de Wuhan, região central da China. Um surto de síndrome respiratória aguda grave se estabelecia, vinculado ao Mercado Atacadista de Frutos do Mar de Huanan (Sul da China) usando um mecanismo de vigilância para "pneumonia de etiologia desconhecida", permitindo a identificação do novo patógeno 2019$\mathrm{nCOV}^{1}$.

Sabe-se que o vírus tem alta transmissibilidade e provoca uma síndrome respiratória aguda de casos leves em cerca de 80\% dos casos. Muitos pacientes serão assintomáticos e poderão ser monitorados em suas próprias residências, porém ainda não há estudos aprofundados sobre a história natural, nem medidas de efetividade para o manejo clínico dos casos de infecção humana pela Covid-19 e políticas ideais para o enfrentamento da pandemia².

Tendo a Atenção Primária (AP) como um conjunto de ações de saúde, no âmbito individual e coletivo, que abrange além da promoção, proteção da saúde, prevenção de agravos, diagnóstico, tratamento, reabilitação, redução de danos e manutenção da saúde, podendo ela ser utilizada como uma potente articulação no manejo clínico de várias doenças ${ }^{3}$.

Pelo caráter de importância na saúde coletiva, as políticas públicas demonstram 
um conjunto de disposições que ajudam nas medidas e procedimentos para a orientação política do Estado e regulam as atividades governamentais relacionadas às tarefas de interesse público ${ }^{4-5}$.

O fato de as decisões serem submetidas às capacidades institucionais a eles disponíveis, de modo que suas metas possam ser alcançadas, facilita sua aplicabilidade, pois não envolve apenas decisões de atores governamentais, mas de decisões factíveis, que se enquadram com o ambiente institucional circundante ${ }^{6}$.

Partindo desse pressuposto, no enfrentamento da Covid-19 é importante que essas ações sejam realizadas por meio do exercício de práticas de cuidado e gestão, sob forma de trabalho em equipe, dirigidas a populações de territórios definidos, considerando a dinamicidade existente no território em que vivem essas populações ${ }^{3}$. Com atualizações frequentes, o Protocolo de Manejo Clínico do Coronavírus (Covid-19) Atenção Primária à Saúde² veio nortear essas ações.

O monitoramento realizado pelas equipes por meio desse protocolo deve auxiliar no manejo das necessidades de saúde de maior frequência e relevância em seu território, observando critérios de risco, vulnerabilidade, resiliência e o imperativo ético de que toda demanda, necessidade de saúde ou sofrimento devem ser acoIhidos ${ }^{3}$.

A Atenção Primária tem um papel importante na vigilância desses casos, pois oferece um atendimento resolutivo, mantendo a longitudinalidade e a coordenação do cuidado em todos os níveis de atenção à saúde². Sendo assim, o trabalho teve como objetivo mostrar o processo e o resultado do trabalho desenvolvido pela Atenção Primária no monitoramento de pacientes suspeitos em Ponta Grossa, Paraná de março a maio de 2020.

\section{MÉTODO}

Tratou-se de uma pesquisa exploratória, descritiva, com o objetivo de mostrar o processo e o resultado do trabalho desenvolvido pela AP no monitoramento de 
pacientes suspeitos de Covid-19 no município de Ponta Grossa, Paraná no período entre março e maio de 2020.

Ponta Grossa é um município brasileiro do Estado do Paraná, sendo o quarto mais populoso, com 351.736 habitantes, conforme estimativa do Instituto Brasileiro de Geografia e Estatística (IBGE) publicada em 20197. Conta com 80 equipes de Estratégia Saúde da Família (ESF), o que condiz com 85\% de cobertura, distribuídos em 54 Unidades de Saúde envolvendo 1.100 colaboradores diretos.

A pesquisa se deu por meio da investigação de como são realizados os registros dos pacientes monitorados por sintomas respiratórios, o fluxo seguido por essas equipes da AP e a forma como os profissionais da saúde realizam a orientação e monitoramento.

\section{RESULTADOS E DISCUSSÃO}

O primeiro caso na cidade foi confirmado em 21 de março, onde o paciente teve histórico de viagem ao exterior. Durante a pandemia, Ponta Grossa adotou estratégias entre março e abril de 2020, desde o total fechamento do comércio através de decretos municipais no dia 23 de março, reabertura gradual do comércio dia 06 de abril com esquema de rodízio e horário de funcionamento no comércio reduzido, restrições em mercados, academias e igrejas, além do uso obrigatório de máscara ${ }^{8-10}$.

A elevada infectividade do coronavírus, ausência de imunidade prévia na população e a inexistência de vacina fazem com que o crescimento do número de casos seja exponencial, se não forem tomadas medidas para deter sua transmissão ${ }^{11}$.

Assim, são indicadas intervenções não farmacológicas (INF), que incluem medidas a toda população, como lavagem das mãos, etiqueta respiratória, distanciamento social, arejamento e exposição solar de ambientes, limpeza de objetos e superfícies, restrição ou proibição ao funcionamento de escolas, universi- 
dades, locais de convívio comunitário, transporte público, além de outros locais onde há aglomeração de pessoas ${ }^{12}$.

Tais medidas, quando adotadas no início de um período epidêmico, auxiliam na prevenção da transmissão, na diminuição da velocidade de espalhamento da doença e, consequentemente, contribuem para achatar a curva epidêmica ${ }^{13}$. A falta de estudos que comprovem essa afirmação no enfrentamento da pandemia pelo Covid-19 contribui para a necessidade de avaliação de sua efetividade $^{14}$.

Entre os 91 municípios com mais de 300 mil habitantes do Brasil, a cidade encontrou-se entre os dois municípios brasileiros, juntamente de Pelotas no Rio Grande do Sul, sem óbitos pelo Covid-1915. A cidade paranaense teve até final de maio o registro de 77 casos confirmados, dos quais 49 recuperados e 1.621 casos monitorados ${ }^{16}$. O panorama dos casos é visualizado ${ }^{17}$ conforme o Quadro 1.

Quadro 1. Panorama geral de casos de Covid-19 até 31 de maio de 2020.

\begin{tabular}{|c|c|c|c|c|}
\hline $\begin{array}{c}\text { Casos } \\
\text { Covid-19 }\end{array}$ & $\begin{array}{c}\text { Mundo } \\
\text { Fonte: OMS }\end{array}$ & $\begin{array}{c}\text { Brasil } \\
\text { Fonte: MS }\end{array}$ & $\begin{array}{c}\text { Paraná } \\
\text { Fonte: SESA }\end{array}$ & $\begin{array}{c}\text { Ponta Grossa } \\
\text { Fonte: FMS }\end{array}$ \\
\hline Casos & 5.934 .936 & 498.440 & 4.473 & 77 \\
\hline Óbitos & 367.166 & 28.834 & 181 & 0 \\
\hline
\end{tabular}

Fonte: Elaborado pelo autor (2020) Legenda: OMS (Organização Mundial da Saúde), MS (Ministério da Saúde), SESA (Secretaria de Estado do Paraná), FMS (Fundação Municipal de Saúde).

Além de assumir um papel restritivo em relação a comércio, uso de máscara, distância segura, redirecionamento correto da população entre os hospitais da cidade e Unidades de Pronto Atendimento (UPA), a AP da Fundação Municipal de Saúde adotou um protocolo específico de atendimento, tendo como guia norteador para as estratégias no enfrentamento da pandemia o Protocolo de Manejo 
Clínico do Coronavírus (Covid-19) na Atenção Primária à Saúde, disponibilizada pelo Ministério da Saúde².

O primeiro fluxo de atendimento para sintomático respiratório utilizado dentro das unidades de saúde foi disponibilizado no dia 09 março, contando diariamente com atualizações disponibilizadas pelo Sistema Eletrônico de Informações (SEI).

Esse fluxo correspondia a atender pacientes com sintomas respiratórios que haviam viajado ao exterior, e/ou contato próximo de caso suspeito e/ou caso confirmado de Covid-19 a contar 14 dias anteriores ao aparecimento dos sinais ou sintomas. Atualmente, todo paciente com sintoma respiratório é considerado caso suspeito e monitorado pelas unidades de saúde. Mudanças foram realizadas com o passar do aumento da gravidade gerada e pelo aumento de casos dentro do município.

A partir do dia 21 de maio de 2020, atendendo aos critérios de definição de caso suspeito, o usuário que comparecesse ao serviço com sintoma de tosse seca, dor de garganta ou dificuldade respiratória, acompanhada ou não de febre, deveria ser acolhido e fornecido máscara cirúrgica à pessoa e encaminhá-la para uma área separada ou sala de isolamento. Todo profissional de saúde que fosse atender esse paciente deveria seguir as orientações para o uso adequado do equipamento de proteção individual (EPI).

O objetivo principal dos fluxos é de nortear o manejo e controle da infecção Covid-19, bem como disponibilizar os instrumentos de orientação clínica para os profissionais que atuam na AP a partir da transmissão comunitária de Covid-19 que o Brasil vem sofrendo².

Após avaliação clínica na unidade de saúde, a notificação era realizada através do sistema "Notifica Covid-19" da Secretaria de Estado da Saúde do Paraná (SESA-PR), onde todos do domicílio são monitorados. O mesmo sendo executado por telefone num período de 14 dias corridos, pelas equipes da área de abrangência e da AP, se encerrando apenas quando o paciente se mantiver por 72 horas assintomático, sendo fornecido atestado médico, se necessário, para todos os familiares. 
A AP recebe de toda rede pública e privada notificações de todos os pacientes sintomáticos respiratórios. O acompanhamento desses pacientes é realizado através do preenchimento de uma planilha criada pela gestão da AP do município, por meio do acesso remoto ao Google Drive contendo informações de nome do caso suspeito, comorbidades, data do início dos sintomas, nome e grau de parentesco dos contatos familiares, telefone de contato, idade, dia e horário do monitoramento, sintomas relatados pelo paciente e pelos demais contatos domiciliares.

As orientações realizadas via telefone partem desde rever a sintomatologia ao total isolamento familiar, medidas de higiene com roupas, lavagem das mãos, uso de álcool 70\%, manter o ambiente ventilado, evitar o contato direto entre os familiares, mantendo uma distância segura com o uso de máscara, seguindo o Protocolo de Manejo Clínico da Atenção Primária.

A AP tem como uma das estratégias desenvolver educação em saúde para a população adscrita, como um processo com princípios críticos e reflexivos e metodologia baseada em diálogo, formando atores sociais integrados e participativos, principalmente, nas questões de gestão da saúde ${ }^{18}$. Assim, a educação em saúde pode auxiliar na compreensão das causas dos problemas de saúde da comunidade, bem como na busca de soluções para os mesmos ${ }^{19}$.

Estudo de revisão sistemática apontou evidências para apoiar o uso de máscaras por pessoas doentes para proteger outras pessoas, e de que recomendações de saúde pública para o uso de máscaras com essa finalidade podem ajudar a reduzir a transmissão do vírus influenza ${ }^{20}$. Outro estudo concluiu que uma combinação de INF, incluindo higiene das mãos, uso de máscaras e isolamento de casos ofereceu o maior grau de proteção contra vírus respiratórios ${ }^{14}$.

Como a AP é a porta de entrada na rede de atenção à saúde, esse contato ao usuário já adscrito facilita a troca de informações e o diálogo frequente com o paciente, facilitando a orientação e o monitoramento dessas famílias. Porém, há necessidade de estudos mais aprofundados que mostrem essa realidade no enfrentamento da pandemia pela Covid-19. 


\section{CONCLUSÃO}

As estratégias desenvolvidas pelo município e pela AP demonstram-se importantes no que se refere à baixa contaminação comunitária dentro da cidade no período em estudo, sem registro de óbito pela Covid-19.

É importante destacar que a busca ativa e o monitoramento dessas famílias na prevenção e esclarecimento da população a respeito do Novo Coronavírus são essenciais no combate à doença, demonstrando assim o grande papel da rede primária de atenção à população no enfrentamento da pandemia.

Faz-se necessário mais estudos em relação a esse enfrentamento, e valorização das Unidades de Saúde como porta de entrada para a grande demanda e luta diária na prevenção e promoção em saúde.

\section{REFERÊNCIAS}

1. Li Q, Guan X, Wu P, Wang X, Zhou L, Tong Y, et al. Early Transmission Dynamics in Wuhan, China, of Novel CoronavirusInfected Pneumonia [Internet]. Massachusetts: The New England Journal of Medicine; 2020. [citado em 2020 Mar 26]; 382: 1199-1207. Disponível em: https://www.nejm.org/doi/pdf/10.1056/NEJMoa2001316?articleTools=true.

2. Brasil. Ministério da Saúde. Protocolo de Manejo Clínico do Coronavírus (COVID-19) na Atenção Primária à Saúde. 2020. Brasília: Ministério da Saúde; 2020. Disponível em: http://docs.bvsalud.org/biblioref/2020/05/1095920/20200504 protocolomanejo-ver09.pdf.

3. Brasil. Ministério da Saúde. Política Nacional de Atenção Básica. 2012. Brasília: Ministério da Saúde; 2020. Disponível em: http://189.28.128.100/dab/docs/publicacoes/geral/pnab.pdf.

4. Lucchese PTR, Aguiar DS, Wargas T, Lima LD, Magalhães R, Monerat GL. Políticas públicas em Saúde Pública. São Paulo: BIREME/OPAS/OMS; 2004.

5. Howlett M, Ramesh M. Studying public policy: policy cycles and policy subsystems. 2. ed. Toronto: Oxford University Press; 2003.

6. Oliveira VE. Saúde Pública e Políticas Públicas: campos próximos, porém distantes [Internet]. São Paulo: Saúde soc.; 2016. 25(4):880-894. Disponível em: https://doi.org/10.1590/s0104-12902016172321.

7. IBGE. Instituto Brasileiro de Geografia e Estatística IBGE. Panorama. Estimativa População [Internet]. Disponível em: https://cidades.ibge.gov.br/brasil/pr/ponta-grossa/panorama.

8. Paraná. Decreto n 17.147 de 21 de março de 2020. Dispõe sobre a suspensão de atividades sujeitas à aglomeração de pessoas no âmbito do Município de Ponta Grossa, em complemento aos Decretos Municipais n. 17.077/2020 e 17.144/2020 [Internet]. Ponta Grossa: Diário Oficial do Município de Ponta Grossa; 2020. Disponível em: http://www.pontagrossa.pr.gov. br/files/diario-oficial/2020-03-22-e-23-2796.pdf.

9. Paraná. Decreto n¹7.211 de 06 de abril de 2020. Dispõe sobre a suspensão de atividades sujeitas à aglomeração de pessoas no âmbito do Município de Ponta Grossa, em complemento ao Decreto Municipal n. 17.207/2020 [Internet]. Ponta Grossa: Diário Oficial do Município de Ponta Grossa; 2020. Disponível em: http://www.pontagrossa.pr.gov.br/files/diariooficial/dom-2020-04-06 2807_fechado.pdf. 
10. Paraná. Decreto $n^{\circ} 17.258$ de 22 de abril de 2020. Dispõe sobre a suspensão de atividades sujeitas à aglomeração de pessoas no âmbito do Município de Ponta Grossa, em complemento aos Decretos 17.207, de 03/04/2020, 17.211, de 06/04/2020, 17.242, de 09/04/2020, 17.243, de 09/04/2020 e 17.255/2020 [Internet]. Ponta Grossa: Diário Oficial do Município de Ponta Grossa; 2020. Disponível em: http://www.pontagrossa.pr.gov.br/files/diario-oficial/dom-2020-04-22extra_2819.pdf.

11. Kucharski AJ, Russel TW, Diamond C, Liu Y, Edmunds J, Funk S, et al. Early dynamics of transmission and control of COVID-19: a mathematical modelling study [Internet]. Londres: Lancet Infect Dis.; 2020. 20 (5): 553-558. Disponível em: https://www.thelancet.com/article/S1473-3099(20)30144-4/fulltext.

12. Garcia LP, Duarte E. Nonpharmaceutical interventions for tackling the COVID-19 epidemic in Brazil. [Internet]. Brasília: Epidemiol. Serv. Saúde; 2020. [citado em 2020 Jul 29]; 29(2): e2020222. Disponível em: http://dx.doi.org/10.5123/s167949742020000200009

13. Qualls N, Levitt A, Kanade N, Jegede NW, Dopson S, Biggerstaff M, et al. Community Mitigation Guidelines to Prevent Pandemic Influenza - United States, 2017 [Internet]. [local desconhecido]: MMWR Recommend Rep.; 2017.66 (Nº. RR-1): 1-34. Disponível em: http://dx.doi.org/10.15585/mmwr.rr6601a1.

14. Garcia LP. Uso de máscara facial para limitar a transmissão da COVID-19 [Internet]. Brasília: Epidemiol. Serv. Saúde; 2020. [citado em 2020 Jul 29]; 29(2): e2020023. Disponível em: http://dx.doi.org/10.5123/s1679-49742020000200021.

15. Felix R. Como uma grande cidade do Paraná evitou mortes por Covid-19, até agora [Internet]. Curitiba: Gazeta do Povo; 2020. Disponível em: https://www.gazetadopovo.com.br/parana/grande-cidade-parana-evitou-mortes-covid/.

16. Paraná. Boletim Oficial Municipal. Prefeitura Municipal de Saúde. Ponta Grossa: Fundação Municipal de Saúde; 2020. Disponível em: http://www.pontagrossa.pr.gov.br/files/31.05.2020_15h_-_boletim_municipal.pdf.

17. Paraná. Informe Epidemiológico COVID-19 de 31/05/2020. Governo do Estado do Paraná [Internet]. Curitiba: SESA; 2020. Disponível em: http://www.saude.pr.gov.br/arquivos/File/INFORME_EPIDEMIOLOGICO_31_05_2020.pdf.

18. Brasil. Ministério da Saúde. Conselho Nacional das Secretarias Municipais de Saúde. O SUS de A a Z: garantindo saúde nos municípios. 3. ed. Brasília: Ministério da Saúde; 2009. 480 p.

19. Vasconcelos EM. Educação popular nos serviços de saúde. 3. ed. São Paulo: Hucitec; 1997.

20. Cowling BJ, Zhou Y, Ip DK, Leung GM, Aiello AE. Face masks to prevent transmission of influenza virus: a systematic review [Internet]. Cambridge: Epidemiol. Infect.; 2010. 138(4):449-56. Disponível em: http://dx.doi.org/10.1017/ S0950268809991658.

\section{RECEBIDO: 15/06/2020 \\ ACEITO: 07/10/2020}

